\title{
Development of a Healthy Lifestyle Mobile App for Overweight Pregnant Women: Qualitative Study
}

Ying Lau ${ }^{1}$, RN, PhD; Ling Jie Cheng ${ }^{2}$, BSc (Hons), RN; Claudia $\mathrm{Chi}^{3}$, MBBS, MD, MRCOG; Cammy Tsai ${ }^{4}$, BS; Kai Wen Ong ${ }^{5}$, BSc (Hons); Sarah Su Tin Ho-Lim ${ }^{3}$, MSc, RN; Wei Wang ${ }^{6}$, PhD; Kian-Lee Tan ${ }^{6}$, PhD

\footnotetext{
${ }^{1}$ Alice Lee Centre for Nursing Studies, Yong Loo Lin School of Medicine, National University of Singapore, Singapore, Singapore

${ }^{2}$ Department of Nursing, Khoo Teck Puat Hospital, Yishun Health Campus, National Healthcare Group, Singapore, Singapore

${ }^{3}$ Department of Obstetrics \& Gynecology, National University Hospital, Singapore, Singapore

${ }^{4}$ Department of Rehabilitation, National University Hospital, Singapore, Singapore

${ }^{5}$ Department of Dietetics, National University Hospital, Singapore, Singapore

${ }^{6}$ Department of Computer Science, National University of Singapore, Singapore, Singapore
}

Corresponding Author:

Ying Lau, RN, PhD

Alice Lee Centre for Nursing Studies

Yong Loo Lin School of Medicine

National University of Singapore

Level 2, Clinical Research Centre, Block MD11, 10 Medical Drive

Singapore, 117597

Singapore

Phone: 6566011603

Fax: 6567767135

Email:nurly@nus.edu.sg

\section{Abstract}

Background: Mobile apps are becoming an increasingly ubiquitous platform for delivery of health behavior interventions among overweight and obese perinatal women. However, only a few methodological guidelines on integrating theory, evidence, and qualitative research for their designs are available.

Objective: The aim of this study was to develop a theory-based, evidence-driven, and user-centered healthy lifestyle app targeting overweight and obese multiethnic pregnant women.

Methods: This paper illustrates how intervention development may be enriched with theoretical basis, systematic review, and qualitative study. An individual face-to-face interview was performed to incorporate the user's involvement in the design. These interviews were audiotaped and transcribed. Thematic analysis technique was used for emerging themes.

Results: Integrated concepts of social cognitive theory of self-regulation, self-regulation model, and strength model of self-control were selected as bases of the intervention. Evidence from our systematic review and meta-analysis provided the strongest evidence for the development of intervention. We invited 16 obese or overweight pregnant women to participate in a semistructured interview. The following key themes emerged: content, platform, interactivity, format, and functionality. Apps are a favorable technology platform for healthy diet advice, appropriate physical exercise, and weight management because they are user-friendly and convenient. The app used in this study contains culture-specific, pregnancy-related, and credible contents, including educational, professional and peer support, and self-monitoring domains. The design should include aesthetic appeal, visualized features, and interactive multimedia.

Conclusions: A 3-step process integrating theoretical basis, evidence from systematic review, and research findings from target users can be considered a guide for future app development.

(JMIR Mhealth Uhealth 2018;6(4):e91) doi: 10.2196/mhealth.9718

\section{KEYWORDS}

mobile apps; overweight; obesity 


\section{Introduction}

\section{Background}

With obesity as a worldwide epidemic [1], perinatal overweightness and obesity have been widely considered [1]. Approximately 50\% of women experience excessive gestational weight gain (GWG) [2], which shows a consistent relation to postpartum weight retention [3] and a substantially increased risk of being obese. Perinatal overweightness and obesity are linked to adverse maternal and infant outcomes, including obstetric, intrapartum complications, instrumental delivery, babies who are large for their gestational age, and macrosomia $[2,4,5]$. Unhealthy lifestyle patterns are critical factors influencing perinatal overweightness and obesity [6]. Behavioral change depends on the discontinuation of an unhealthy lifestyle and the formation of a new healthy lifestyle [7]. Pregnancy is a crucial stage to remain healthy for the sake of pregnant women and their unborn baby [8]. Healthy eating during pregnancy is critical, and pregnant women require $2000 \mathrm{kcal} /$ day [9]. To obtain a balanced diet, pregnant women should eat a variety of food, including fruits, vegetables, rice, meat, and milk and its alternatives [9]. With regard to physical activity, pregnant women should walk 10,000 steps a day (4-5 miles, depending on stride length) or do a minimum of 30-min moderate physical activity for 5 to 7 days a week [10]. The Institute of Medicine guidelines recommend a total weight gain during pregnancy based on the prepregnancy body mass index (BMI) as follows: normal, 11.5 to $16 \mathrm{~kg}$; overweight, 7 to $11.5 \mathrm{~kg}$; and obese, 5 to $9 \mathrm{~kg}$ [11]. Consequently, new and effective lifestyle interventions that promote healthy outcomes are necessary.

Mobile apps create new opportunities to set behavioral goals, provide healthy lifestyle counseling, and facilitate self-monitoring of pregnant women's goal-directed behavior [12]. Apps have become increasingly relevant to health care. Apps have been successfully integrated into interventions that target diet, physical activity, and weight management in overweight and obese individuals [13]. Apps also use a tracking system to improve adherence by automatic alert or notification or graphic progress by monitoring devices for reminders and regular interactions. The advantages of using apps include cost-effectiveness, accessibility, and timely delivery to multiple regions and various populations [13,14]. Perinatal women actively use apps to search for pregnancy health-related information, discuss issues with peers, and seek advice from professionals to guide their pregnancy decision making $[15,16]$. Nonetheless, the market for health care apps is considerably fragmented because many of them are designed for highly specific contexts, and they lack theoretical content. A systematic review on quality assessment for apps [17] is varied. Results showed that only 10 studies from 606 articles satisfy the inclusion criteria. According to the quality criteria, the mean score is 5.05 out of 8 [17]. Thus, the development of a quality and evidence-based app for overweight or obese perinatal women is necessary.

Theoretical-based intervention helps guide intervention designers in identifying theoretical constructs to target in an intervention to elicit behavioral change [18]. In addition, theoretical foundation provides guidance on mobile health behavior intervention development [19]. The Template for Intervention Description and Replication (TIDieR) guideline [20] recommended the use of theoretical frameworks in designing interventions. More importantly, theory-informed development facilitates the functionality of an intervention [21]. Evidence from systematic review and meta-analysis is used as a basis to develop recommendations for mobile app development [22]. Systematic review is the reference standard in synthesizing evidence in health care [23]. Moreover, a meta-analytic approach is considered the strongest evidence because of its methodological rigor [24]. User-centered design is a well-established approach to develop a mobile app. This design is strategically important because of its insights on users and their context of use [25]. Advantages of user-based design include the promotion of autonomy, competence, positive emotional experience, and sense of relatedness for users [26]. User-based design focuses on target audience through an iterative design process that engages users in conceptualization, design, and development of an app [27]. User involvement increases appeal and user-friendliness [28]. Target population can select tailored information about their preferred form, which is essential to maximize the acceptability and effectiveness of interventions [26]. User-centered development method can assist in understanding the preference of potential users for content, platform, and format, thereby resulting in a remarkably effective program.

\section{Objectives}

With potential benefits of low cost, high accessibility, and good adherence, this study aims to develop a mobile app (mHELP) for a healthy lifestyle program among overweight or obese multiethnic pregnant women using a theory-based, evidence-driven, and user-centered approach.

\section{Methods}

With regard to mHELP development, we used a 3-step process by integrating theoretical basis, evidence from our systematic review, and research findings from our target users.

\section{Step 1: Theory-Informed Development}

Intervention development by using a theoretical basis can substantially improve health behavior [18]. Hence, mHELP development was based on the integrated concepts of social cognitive theory of self-regulation [29], self-regulation model [30], and strength model of self-control [31]. The social cognitive theory of self-regulation emphasizes the major self-regulative mechanism through the 3 principal subfunctions, including self-monitoring of individual behavior (its determinants and its effects), evaluation of individual behavior in relation to personal standards and environmental circumstances, and affective self-reaction [29]. The self-regulation model focuses on a 5-stage self-regulation, including specification of goals, establishment of commitments to change, physical and environmental management to facilitate pursuit of goals, and execution of self-regulation components to achieve the goal [30]. The strength model of self-control consists of the following components: standard of desirable behavior, motivation to satisfy standards, monitoring of 
situations that achieve the standards, and internal strength to control urges [31]. Self-control is a central function of an individual. This function is an important key to succeed in life [31] because it alters the individual's responses. In particular, self-control aligns an individual with standards and supports the pursuit of a long-term goal. The conceptual framework (as shown in Figure 1) illustrates the relationships between mHELP and health outcomes.

In this conceptual model, self-regulation involves self-awareness of the current overweight and obese status. Awareness can trigger a self-evaluation response, which involves the interpretation of the condition of an individual against a goal or a standard. In addition, a series of responses can be determined after self-evaluation as a result of self-adjustment and self-reinforcement [30] to improve healthy diet pattern, increase physical activities, and obtain appropriate GWG. Consequently, women who participated in using the mHELP app will probably obtain improved maternal and neonatal outcomes.

Figure 1. Conceptual framework.

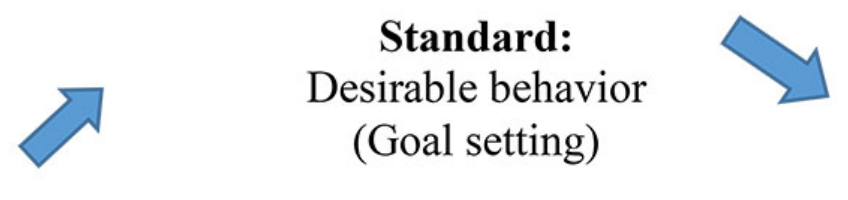

Self-reinforcement: Positive performance will increase self-efficacy and negative performance will motivate to work harder

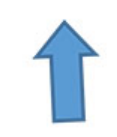

\section{Self-adjustment:}

A serious of responses for adjusting weight, diet, and physical activity

\section{Mobile app for a healthy lifestyle program (mHELP) in overweight or obese multiethnic perinatal women}

Motivation:

Selfawareness

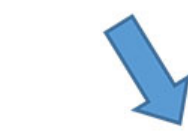

\section{Internal strength: \\ Self-control}

Improve healthy eating pattern

Increase physical activities

Have appropriate gestational weight gain

\section{Self-evaluation:}

Interpretation of weight gain, diet intake, and physical activity against a standard

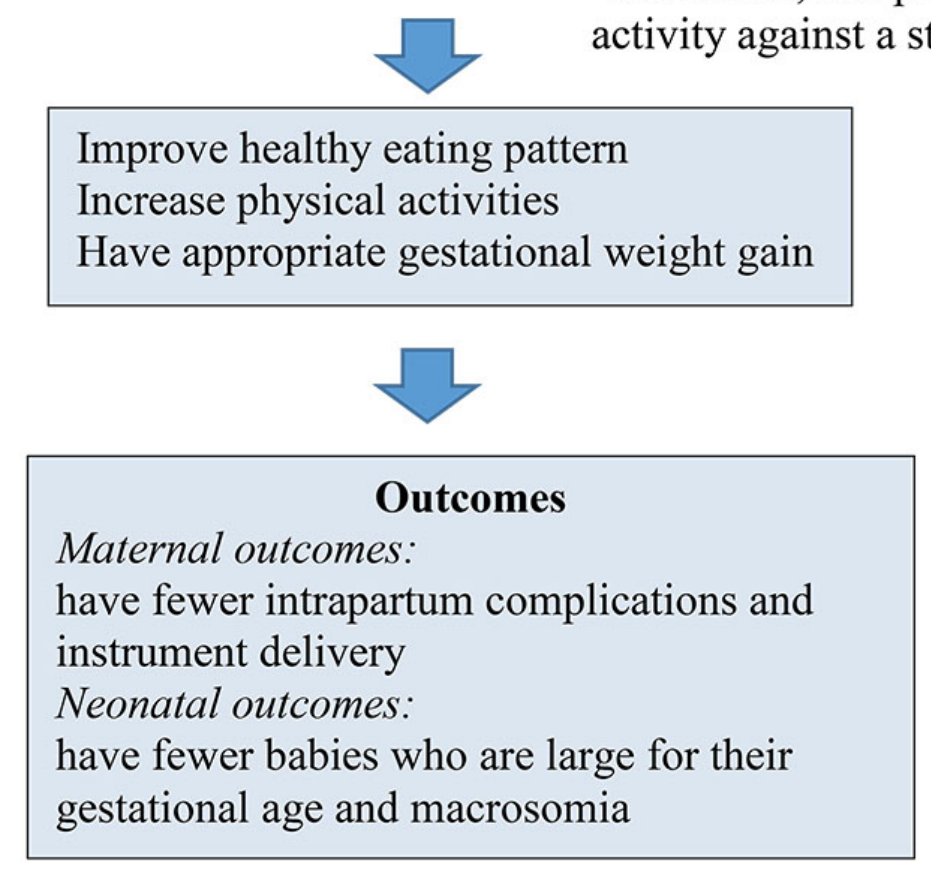




\section{Step 2: Evidence From Our Systematic Review and Meta-Analysis}

To obtain the most significant available evidence to design mHELP, our research team conducted a systematic review and meta-analysis to evaluate the effectiveness of electronic-based (e-based) lifestyle interventions in overweight or obese perinatal women [32]. Seven electronic databases, including the Cumulative Index to Nursing and Allied Health Literature, Cochrane Library, Excerpta Medica database, ProQuest Dissertations and Theses, PsycINFO, PubMed, and Scopus, were searched from their inception to July 13, 2016. We selected 14 randomized controlled trials (RCT) in 17 publications among the 1145 available studies [32]. Our review found that e-based lifestyle intervention is an acceptable approach to limit GWG, lose postnatal weight, increase the moderate and vigorous physical activity, and reduce the calorie intake after intervention [32].

Different e-based delivery formats were observed in 14 selected RCTs; these formats include app [12], website [33], the internet [34], email [35], short message services [36], computers [37], and interactive videos [38]. Physical activity, diet, and weight management are essential components in designing healthy lifestyle interventions [32]. Promising strategies play key roles in promoting healthy lifestyle. These strategies include setting of behavioral goals, undergoing lifestyle counseling or skills training, regular self-monitoring, and receiving reinforcement through feedback from health care professionals. Intervention starts in the first trimester of pregnancy until postpartum periods to broaden the beneficial effect of intervention [39]. E-based platforms incorporating in-person and phone session for professional consultation are effective in reducing GWG [32] because synchronous interpersonal interactions may be beneficial in improving the effectiveness of the intervention [40]. Social networking among peer support is vital in promoting healthy behavior [41]. Monitoring device for physical activity [42], image-assisted dietary assessment [43], and anthropometric measures for nutritional status [44] are considered accurate outcome measurements. Table 1 summarizes the suggested recommendations from our systematic review [32]. These recommendations can guide our study in exploring the subsequent step in designing a lifestyle program for overweight and obese perinatal women.

\section{Step 3: Use of Qualitative Research to Inform User-Centered Design}

A qualitative methodology involving end users in the design process was used [25]. Qualitative research method is important because it provides useful insights for initial elicitation to design and develop mHELP [45]. A user-centered approach helps explore the needs and preferences of content, platform, and format of mHELP among overweight or obese multiethnic perinatal women [25]. The outcomes of this qualitative research may facilitate in tailoring the intervention, thereby increasing its acceptability and effectiveness.

\section{Sample and Setting}

We purposively recruited 16 participants among multiethnic overweight and obese pregnant women in 2 outpatient clinics in a hospital, which is a 1160-bed, university-affiliated hospital that serves more than 670,000 outpatients and 49,000 inpatients. This hospital provides comprehensive obstetric care for different demographic and socioeconomic groups in Singapore, with a delivery rate of 3233 deliveries/year. The inclusion criteria for participating in the study included pregnant women aged $\geq 21$ years with prepregnancy BMI of $\geq 25.0 \mathrm{~kg} / \mathrm{m}^{2}$ and having a singleton uncomplicated pregnancy at no more than 30 weeks of gestation.

\section{Data Collection}

Data collection was conducted from July 2016 to January 2017 after obtaining approval from the Domain Specific Review Board (Reference No: NHG DSRB 2016/00654). A research assistant approached the target pregnant women during antenatal clinic visits, and eligibility screening was performed in a private area. Participants were informed about the purpose of the study, and information sheet was given. This initial contact was followed up by a telephone interview to establish their interest and consent to participate. Informed written consent was obtained, and participants' profiles were collected. A qualified research assistant, who was trained to conduct qualitative face-to-face interviews, carried out all individual interviews to ensure a high level of consistency. A semistructured interview guide (Textbox 1) with open-ended questions was used to explore the needs and preferences of participants in terms of the content, platform, and format for a healthy lifestyle intervention. 
Table 1. Recommendations from the systematic review and meta-analysis.

\begin{tabular}{ll}
\hline Aspect & Recommendations \\
\hline Component & Physical activity, diet, and weight management \\
Period & First trimester to postnatal period \\
Platform & E-based platform incorporating in-person and phone session \\
Strategies & $\begin{array}{l}\text { Setting behavioral goals, undergoing lifestyle counseling or skill training, } \\
\text { regular self-monitoring, and receiving reinforcement through feedback }\end{array}$ \\
Outcome measures & $\begin{array}{l}\text { Monitoring device for physical activity, image-assisted dietary assessment } \\
\text { for dietary intake, and anthropometric measures for nutritional status }\end{array}$ \\
Interactivity & $\begin{array}{l}\text { Online peer discussion forum, texting, email, or phone for professional } \\
\text { consultation }\end{array}$ \\
Functionality & $\begin{array}{l}\text { Graphs for progress report, navigation, search feature, goal tracking, noti- } \\
\text { fication or reminder, and link to remote device }\end{array}$ \\
\hline
\end{tabular}

Textbox 1. Semistructured interview guide for face-to-face individual interview.

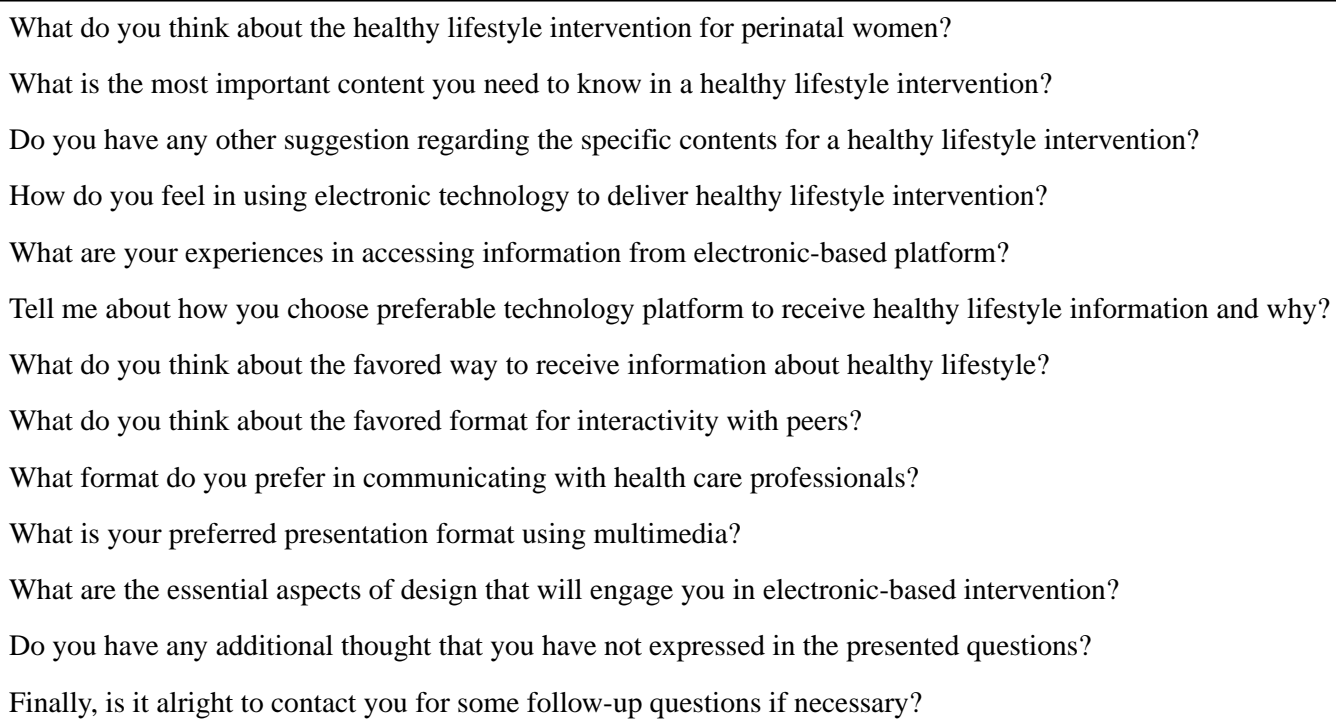

This interview technique allowed participants to express their answers freely in their own words. The interview questions and prompt were developed according to a framework for a qualitative semistructured interview guide [27]. All interviews were conducted in a private room at the convenience of the participant, either before or after the scheduled follow-up at 2 outpatient clinics. The interview lasted for 20 to $40 \mathrm{~min}$. All interviews were audio-recorded, and field notes were taken. We provided each participant $\$ 20$ (Singapore dollar) as a token of appreciation for their time.

\section{Data Analysis}

Descriptive statistics summarized the participants' characteristics. Data were audio-recorded and transcribed verbatim. We used thematic analysis following the methods of Braun and Clarke [46]. This method was selected because of its flexibility, freedom from specific theoretical framework, ability to explore a rich set of data, and identification and analysis of repeated themes [46]. This analytic process is a theoretically flexible method with 6 recommended steps [46], including (1) familiarization with data by reading and rereading the transcripts, (2) initial coding by systematically identifying and naming units of meaning with codes, (3) searching for themes among the initial codes according to data patterns, (4) reviewing themes by organizing the data that may best fit together as subthemes, (5) defining and naming final major themes, (6) producing the report. The themes identified on a semantic level were closely linked to the data using an inductive approach. Constant comparative analysis was performed to iterate the variation between theme occurrences across different participants [47]. Thematic saturation was achieved at the 16th interview as determined by 2 research team members (YL and LJC) during concurrent analysis. Illustrative verbatim quotations were selected to support the validity of data generation.

\section{Methodological Rigor}

Credibility, dependability, confirmability, and transferability establish the methodological rigor [48]. To ensure the credibility of data sources, prolonged engagement and member checks were used. We repeatedly viewed the audio recording, transcription, and field notes for accuracy. We sent the transcriptions to the participants to validate the information through the member-checking procedure [49]. Dependability was achieved by auditing. Audit trails were developed using reflexive memos and codebooks throughout the research process to keep track of any bias and assumption [50]. For 
confirmability, we involved coresearchers in the analysis. Two research members (YL and LJC) independently participated in a multiphase process, including initial coding, theme development, review, and definition [46]. Two members verified this process for accuracy and appropriateness. Any discrepancy was discussed and resolved for consensual validation. Our research team facilitated transferability by providing details of demographic and obstetric descriptions and using relevant quotations from different participants [48].

\section{Results}

\section{Overview}

We invited 20 eligible women, but 4 women refused to join because of lack of time and planning to deliver in another hospital. A total of 16 eligible women (response rate=80\%) agreed to participate; of these, 8 were recruited from a general outpatient clinic, and 8 were from a private outpatient clinic. Table 2 presents a summary of the demographics and obstetric characteristics of all participants. The participants' BMI ranged between $25 \mathrm{~kg} / \mathrm{m}^{2}$ and $38 \mathrm{~kg} / \mathrm{m}^{2}$. Majority of the participants were married and with complete tertiary education.

After a 6-step thematic analysis, 5 key themes related to the domains of content, platform, interactivity, format, and functionality emerged. These 5 themes captured the meaning of narratives offering contextual insights into the development of mHELP. Summaries of the key themes, subthemes, and recommendation are presented in Table 3.

Table 2. Characteristics of interview participants $(\mathrm{N}=16)$.

\begin{tabular}{|c|c|}
\hline Characteristics & $\mathrm{n}(\%)$ \\
\hline \multicolumn{2}{|l|}{ Ethnicity } \\
\hline Chinese & $3(19)$ \\
\hline Malay & $6(37)$ \\
\hline Indian & $4(25)$ \\
\hline Burmese & $1(6)$ \\
\hline Hispanic & $1(6)$ \\
\hline Bangladeshi & $1(6)$ \\
\hline \multicolumn{2}{|l|}{ Age, in years } \\
\hline $25-34$ & $11(69)$ \\
\hline $35-45$ & $5(31)$ \\
\hline \multicolumn{2}{|l|}{ Marital status } \\
\hline Married & $15(94)$ \\
\hline Divorced & $1(6)$ \\
\hline \multicolumn{2}{|l|}{ Education } \\
\hline Degree and above & $11(69)$ \\
\hline Diploma and below & $5(31)$ \\
\hline \multicolumn{2}{|l|}{ Employment status } \\
\hline Full time or part time & $11(69)$ \\
\hline Unemployed or housewife & $5(31)$ \\
\hline \multicolumn{2}{|l|}{ Body mass index, in $\mathrm{kg} / \mathrm{m}^{2}$} \\
\hline 25-30 (overweight) & $10(62)$ \\
\hline 31-38 (obese) & $6(38)$ \\
\hline \multicolumn{2}{|l|}{ Number of pregnancies } \\
\hline 1 & $7(44)$ \\
\hline $2-4$ & $9(56)$ \\
\hline \multicolumn{2}{|l|}{ Number of babies } \\
\hline 0 & $7(44)$ \\
\hline 1 & $5(31)$ \\
\hline $2-3$ & $4(25)$ \\
\hline
\end{tabular}




\section{Theme 1: Content}

\section{Subtheme 1.1: Culturally Tailored and Specific to Pregnancy}

Most participants said that they were highly attracted to engage in the intervention if it can provide them with a culture-specific diet plan. Some participants expressed their opinions as follows:

Maybe something related to like different culture.

Because I am Malay...maybe the meal plan should be customized to race or culture so that I do not need to adapt diet from other culture. [Participant 7]

Whatever I can search pregnant-related information from the internet is mostly developed by the western countries...so the baby size, the body weight of the baby and mother is very much westernized. So I think maybe I should cater to Asian mother and the baby. [Participant 3]

Some participants wanted pregnant-specific healthy lifestyle. They stated:

I like all provided information is pregnancy-based, any suggested activities should be pregnancy-friendly. [Participant 1]

We are pregnant so it is slightly different from the normal people...so I think information will be very helpful only for the pregnant women. [Participant 3]

Table 3. Key themes, subthemes, and recommendations. SMS: short message service.

\begin{tabular}{|c|c|}
\hline Themes & Recommendation \\
\hline \multicolumn{2}{|l|}{ Content } \\
\hline Culturally tailored and specific to pregnancy & $\begin{array}{l}\text { Culture-specific, pregnancy-related, and credible educational support for } \\
\text { diet, exercise, and weight advices for perinatal women }\end{array}$ \\
\hline \multicolumn{2}{|l|}{ Multicomponent } \\
\hline \multicolumn{2}{|l|}{ Credibility } \\
\hline \multicolumn{2}{|l|}{ Platform } \\
\hline Use of mobile app & Mobile app is used in a user-friendly design \\
\hline \multicolumn{2}{|l|}{ Convenient and user-friendly } \\
\hline \multicolumn{2}{|l|}{ Interactivity } \\
\hline Flexible communication with health care professionals & $\begin{array}{l}\text { Professional support via email, SMS text messaging, and hotline for indi- } \\
\text { vidual consultation }\end{array}$ \\
\hline Importance and value of peer support & Peer sharing and support using online peer discussion forum \\
\hline \multicolumn{2}{|l|}{ Format } \\
\hline Aesthetic appeal & $\begin{array}{l}\text { Interactive multimedia, including video, animations, game, and Web-based } \\
\text { quiz }\end{array}$ \\
\hline \multicolumn{2}{|l|}{ Visualized features } \\
\hline \multicolumn{2}{|l|}{ Interactive multimedia } \\
\hline & External link for further resources, such as websites, text, news, and articles \\
\hline \multicolumn{2}{|l|}{ Functionality } \\
\hline Self-monitoring for individualized goal setting & Graphs for progress report using tracking and notification \\
\hline Progress monitoring & Incorporated with remote device (pedometer) \\
\hline Regular update & Easy navigation and automatic update \\
\hline
\end{tabular}

\section{Subtheme 1.2: Multicomponent Content}

The participants also suggested that multicomponent content possesses a key role in designing the content of intervention according to different educational needs. Intervention components should highly emphasize on appropriate exercises, dietary advices, and weight management. Participants' preference is illustrated in the following statements:

Usually it's very helpful to know like which type of food, how many calories or what kinds of food I can eat for controlling my weight. [Participant 5]
I actually don't know to what extend I can exercise. What is suitable exercise for different period of pregnancy?...How much exercise per day or per week that I should do that is good for my body weight? [Participant 7]

\section{Subtheme 1.3: Credibility}

Participants raised the issue about the intervention's credibility. They felt comfortable with obtaining information from reputable sources, such as their doctors, nurses, university, or hospital, rather than from unknown sources regarding the authorship or institution of origin. Two participants shared their experiences: 
Some websites do not provide any information about themselves and I don't believe it. I like information from doctors or nurses that I can trust. [Participant 7]

I think if information source comes from hospital or university, accuracy is the first thing I would expect and credibility is already there. [Participant 8]

\section{Theme 2: Platform}

\section{Subtheme 2.1: Use of Mobile App}

Most participants expressed that the use of mobile app is a preferable technology platform. The main advantage of using mobile apps is that it is handy and easy to navigate; it can be accessed anywhere at any time. Participants stated the following:

Personally, I tried out a number of pregnancy apps. I found mobile phone is quite handy and mobile app is easy to download. [Participant 4]

Nowadays, everybody uses mobile phone and everything is on the app. App likes a one-stop place for everyone and I can access anywhere at any time. App can provide hyperlinks that I could click on if I wanted to. [Participant 12]

\section{Subtheme 2.2: Convenient and User-Friendly}

The participants mentioned that the platform should be convenient and user-friendly. They stated:

For me, user-friendly is very important because I like a simple, user-friendly and direct way to see things. I want to go straight down the page and get where I want to go. [Participant 8]

User-friendly is good! That we can easy to use to read, key in, and find things. [Participant 14]

\section{Theme 3: Interactivity}

\section{Subtheme 3.1: Flexible Communication With Health Care Professionals}

Participants agreed that Web-based consultation is crucial to provide helpful advice and suggestion. Participants also tend to interact with health care professionals in a flexible manner through different delivery modes. Participants stated:

I want some experts provide professional advices on appropriate exercise and balanced diet so online consultation should be very helpful. [Participant 11]

I might not be able to make the interaction in person because of transportation issue or I might have my work. It's easier for me to consult them through phone, email or Skype if there is any misunderstanding I can ask for clarifications. [Participant 7]

\section{Subtheme 3.2: Importance and Value of Peer Support}

Participants felt that intervention can potentially provide professional support and emphasized the importance of peer support from online discussion forums. They stated:
It is a good to have our online discussion forums with other pregnant women and we can exchange our views or maybe even get some information and some help through online discussion forum. [Participant 2]

I enjoy online chatting with other pregnant women and it can offer tips and suggestions for my pregnancy. Honestly, I want reassurance about my status during pregnancy and it means a lot to me if someone's back up. [Participant 11]

\section{Theme 4: Format}

\section{Subtheme 4.1: Aesthetic Appeal}

Most of the participants pointed out that the aesthetic appeal of the intervention is a major determinant in their usage of the app. Thus, considering a colorful and attractive interface is essential. Two typical answers are shown below:
Of course bright color as it can attract people to look at it...so it is easy to catch attention and facilitate learning. [Participant 3]
I prefer colorful design such as pastel color. Generally, I relate pastel color to baby. Pink for girls and baby blue for boy. [Participant 4]

\section{Subtheme 4.2: Visualized Features}

Visual information regarding the quantity and type of food to eat during pregnancy is also an important consideration. Visualized features improve the ability of women to grasp their progress and recognize their food intake. Participants commented:

\section{I like more visuals and graphics for knowing my condition. [Participant 3] \\ It is really good to show me what a standard serving size is supposed to look like because I want to see it. [Participant 10]}

\section{Subtheme 4.3: Interactive Multimedia}

The general preference of the participants is the use of interactive multimedia, including short video scripts, graphs, photos, Web-based quizzes, and animation, to make the app highly engaging. Some participants commented that they appreciate the option of using different multimedia to engage in the intervention, as echoed in the quotes below:

\section{Short video for suggested exercise, health tips and the recommended diet would be interesting and useful. [Participant 6] \\ Personally, I prefer more photos, animation and graphics. Ifeel online quiz is an awesome part. Once I read the information, then I just check whether I have understood it correctly through quizzes. [Participant 8]}

\section{Theme 5: Functionality}

\section{Subtheme 5.1: Self-Monitoring for Individualized Goal Setting}

The participants recognized that each pregnancy presents distinct challenges. They want content in terms of healthy lifestyle 
tailored to their needs and expectations. A general consensus by the respondents indicated that the program should possess functions that will allow self-monitoring. Two participants shared their ideas as follows:

I would like to have some graphs for monitoring my diet, activities and weight that is good. [Participant 7]

I can insert my personal information for the doctor to see using the graph or the chart to monitor how well do I manage, the things to do prior to the next appointment so that the doctor can review. [Participant 10]

\section{Subtheme 5.2: Monitoring the Progress}

Participants felt that the contents should motivate and remind the users of their progress. The participants expressed a desire to monitor their food intake, physical activities, and weight. This result corresponded to other statements from other participants, as evidenced by the quotes below:

Ineed to motivate and remind myself. It is really good if the intervention can give me notification to keep on track. [Participant 4]

Hoping to have simple diet, weight and exercise device or tool to monitor my progression and a routine pop-up message is helpful for reminding me. [Participant 14]

\section{Subtheme 5.3: Regular Update}

Given the rapid change in knowledge, regular updating of content is considerably important. Participants requested for a regular update on the app as a part of the functionality of the intervention. Two participants commented as follows:

I like to read current research and know the latest perinatal diet advice or suggested exercise. [Participant 7]

It will be good if weekly update from intervention to get more updated knowledge. [Participant 8]

\section{Translating Theory, Evidence, and User Needs Into Intervention}

On the basis of the results from the theoretical basis and recommendations of our systematic review and qualitative research findings with target users, the program content, platform, and format of mHELP were formulated. A multidisciplinary research team was formed. This team included a computing expert, a professional app designer, an obstetrician, a dietician, a physiotherapist, a nursing specialist, and a researcher to design mHELP. mHELP aims to improve the neonatal and maternal outcomes in overweight or obese multiethnic perinatal women. To design a highly automated mobile app with a user-friendly interface, mHELP's user interface and features were designed by a computer expert and a professional app designer. The app can be preinstalled on phones and delivered as a Web app to provide online colorful features, such as social networking and tracking within a Web browser. The software program was used in multiple mobile platforms, such as Android and iOS (iPad and iPhone). A visual graphing function was designed to allow the user to configure their starting weight, food intake, and physical activity throughout the pregnancy. Real-time feedback and automatic notification were also developed in the app. Our research team will incorporate social functionality in a large, open-source software hosting service GitHub that makes a developer's identity, so that mHELP can be publicly visible across a wide community.

mHELP content consists of healthy diet advice, appropriate physical exercise, and weight management starting from 12 weeks of gestation to 6 months postpartum, as shown in Textbox 2. Users can access mHELP in their own time and at their own pace. They can also revisit the contents without time limitation. This app contains culture-specific, pregnancy-related, and credible contents, including educational, professional and peer support, and self-monitoring domains. Educational support aims to motivate participants by determining the importance of changing their diet and physical activity to maintain appropriate weight gain. Peer support aims to mediate interaction of participants with one another using a pseudonym via online peer-to-peer communities, which are used to mobilize and raise collective awareness [51]. Professional support aims to achieve adherence and enhance healthy lifestyle knowledge through asynchronous and synchronous feedback [52]. Self-monitoring aims to encourage users to self-regulate their lifestyle behavior according to their goals and reinforce any change made. If the result is below or above the range, then the system provides a notification through the app. The user story box and mock-up screenshots of mHELP are illustrated in Figures 2 and 3, respectively, and its description is presented in Textbox 2. 
Textbox 2. mHELP description.

- Technology platform: mHELP is a responsive Web app that can be used on personal computers, tablets, or smartphone devices through common Web browsers and operating systems

- User interface: colorful and user-friendly

- Content: multicomponent with 4 domains

\section{Domain 1: Educational support}

1. Content

- Content is obtained from national guidelines, theory, evidence, and experts.

- Information is updated and contents are constantly updated to maintain the user's interest

- Mobile app incorporating in-person communication via Skype, email, short message services (SMS), and phone session

2. Pregnancy-related physical activity

- $\quad$ Safety issue for physical activity during perinatal period

- Aerobic exercise: (1) walking, (2) swimming, and (3) yoga

- Strength training: (1) foot and ankle exercise, (2) calf stretch, (3) pelvic floor exercise, and (4) pelvic tilting

3. Culture-specific healthy diet

- Appropriated weight gain

- Healthy diet plan in Chinese, Indian, and Malay styles

- Additional nutrient needs during pregnancy

- Pregnancy food myths

4. Format and security

- Interactive multimedia, including short video scripts, animations, illustrations, games, and online quizzes

- External link to credible resources

- Customizable details and user settings

- Password-protected to ensure online security

- User portal via a privacy compliant-shared record platform

\section{Domain 2: Professional support}

1. Content

- Feedback from health care professionals (obstetrician, dietician, physiotherapist, and nurse) will improve the compliance and knowledge.

2. Interactivity

- Online forum for group consultation

- Users can contact through email, SMS, and/or telephone for individual advice from experts if needed

- Content-related questions are addressed promptly

\section{Domain 3: Peer support}

1. Content

- $\quad$ Provide a forum for the participants to converse with one another

2. Interactivity

- Online peer-directed forum for sharing and support

- User can see how others responded to the poll questions about healthy lifestyle

- User can read posts by other participants; they can also contribute responses in the online forum

Domain 4: Self-monitoring 
1. Individualized goal setting

- Physical activity: engaging in 30 min of moderate to vigorous physical activity at least 5 days per week or walking 10,000 steps a day

- Dietary: improving or maintaining the nutritional quality of their diets by consuming 5 servings of fruits and vegetables per day based on the $2000 \mathrm{kcal} / \mathrm{day}$ requirement and avoiding excess sugar and fat intake and emotional eating

- Weight: following Institute of Medicine-recommended weight gain during pregnancy

2. Functionality

- Users set individualized behavioral goals, and goal achievement triggers onscreen congratulatory feedback. Generate a list of goals in the form of action plan per week and archived goal content

- Offer visualization tools, such as graphic progress chart and food image-assisted dietary advice or assessment

- Navigation, tracking, and notification

- $\quad$ Synchronized with remote monitoring devices (pedometer)

- $\quad$ Tailored messages displayed on app and delivered via SMS or email according to the user's preference

- Regularly updating app with current content

- Auto-update function for app system 
Figure 2. User story box of the mHELP.

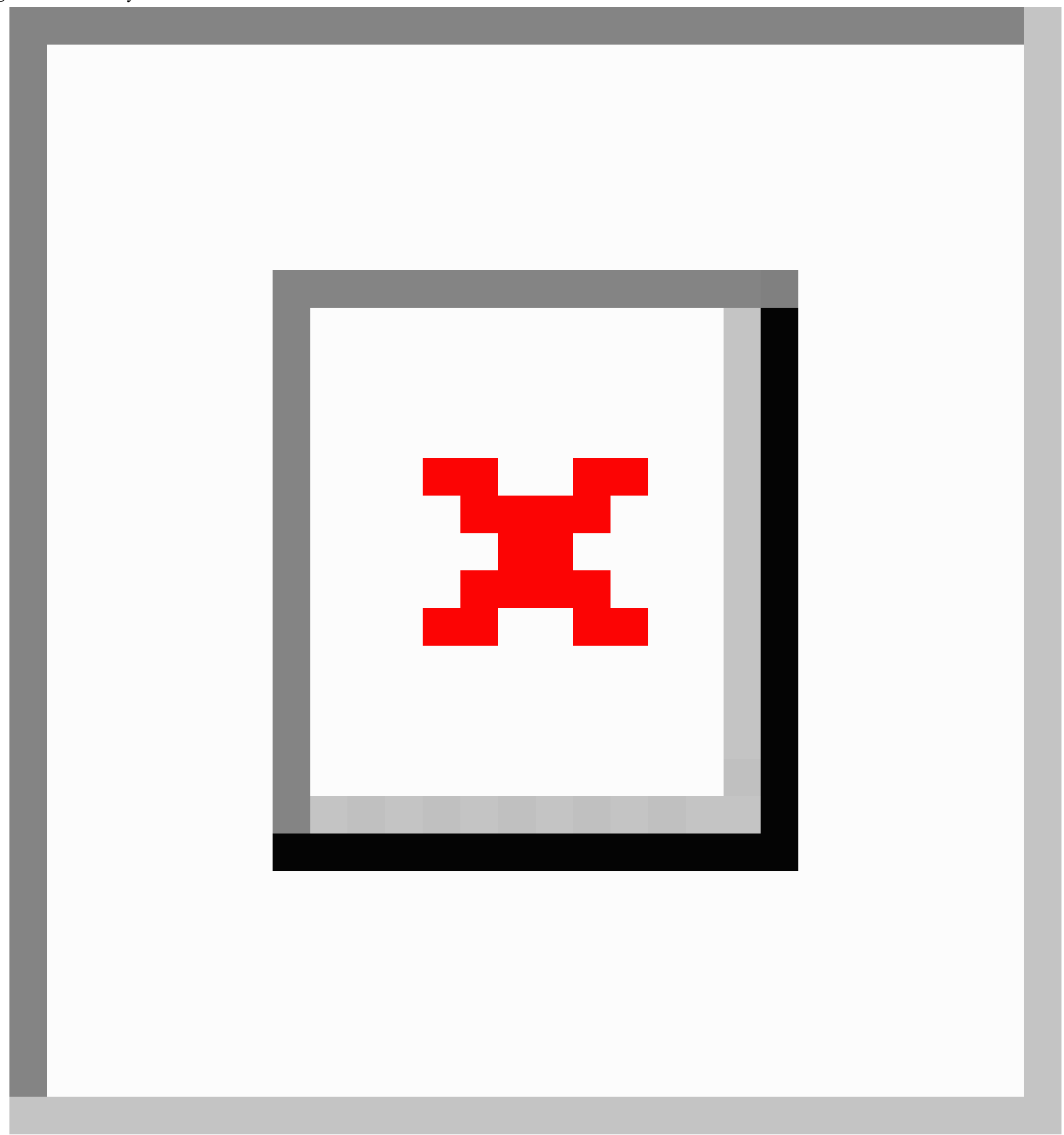


Figure 3. Mock-up screenshots of mHELP.

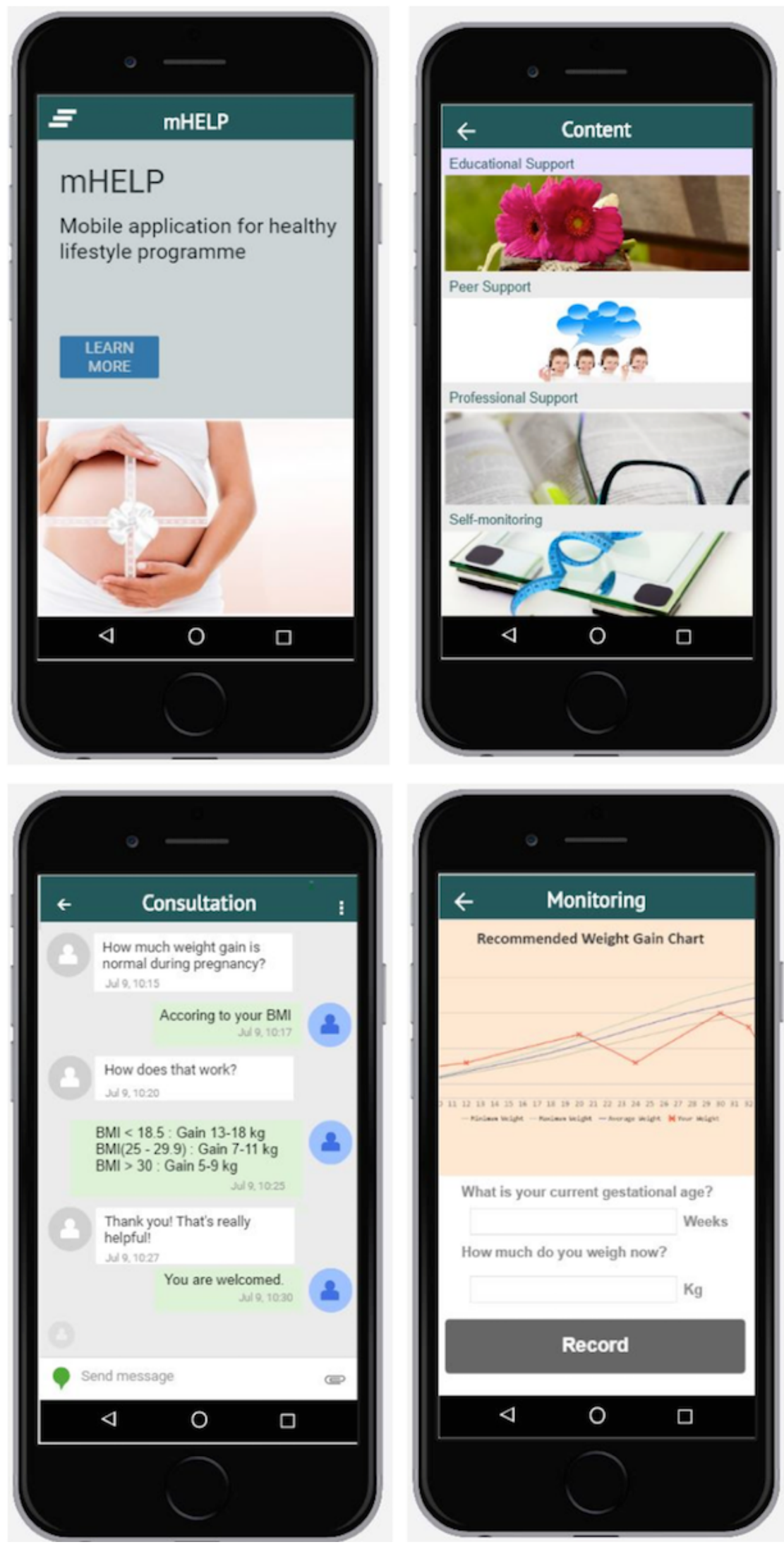




\section{Discussion}

\section{Overview}

To our knowledge, this study is the first to use a theory-informed, evidence-driven approach, and user involvement in developing a mobile app for a healthy lifestyle program in overweight or obese multiethnic perinatal women. We provide the description of a 3-step process by integrating theoretical basis, evidence from our systematic review, and research findings from target users. In addition, multidisciplinary research team was formed to provide expert advice and contribute practical and clinical considerations in designing mHELP.

\section{Principal Findings}

We developed a theory-based intervention following the TIDieR intervention guideline [20]. Integrated concepts from social cognitive theory of self-regulation [29], self-regulation model [30], and strength model of self-control [31] were used to provide theoretical guidance on the app's development. Our systematic review and meta-analysis synthesized the most remarkable evidence using 14 RCTs [32] to provide valuable recommendations on component, period, platform, strategies, outcome measures, interactivity, and functionality of the intervention. With consideration that meta-analysis is the strongest and highest quality of evidence [24], mHELP suggested an evidence-based design. Furthermore, our qualitative study among potential users builds on our previous systematic review and meta-analysis [32] to explore the needs and preferences to design a tailored intervention. The use of a qualitative approach to elicit the user's views during intervention development is considered a good practice [45]. This user-centered design is tailor-made to the end-user perspectives because it can ensure that the app is desirable and suitable for end users [26] by selecting preferable content, technology platform, user interface, interactivity, and functionality.

Themes that emerged from our qualitative study suggested that culturally tailored, pregnancy-specific, multifaceted, and credible contents are particularly important. Given the multiethnic groups in Singapore, we considered to make the content culturally sensitive by matching health information to the observable characteristics of a target group. This effort involves translations to Chinese, Malay, and Indian using pictures of pregnant women with various ethnic backgrounds and using food items familiar to and preferred by pregnant women in those cultures. Users are likely to engage actively in mHELP if they perceive that the intervention is relevant to pregnancy. Multifaceted contents provide a first exposure to educational information in virtual learning environment to satisfy educational needs. Educational support may motivate active learning; in addition, the overall concept is that the users take ownership of their learning [53]. Design format should include aesthetic appeal, visualized features, and interactive multimedia. Multimedia features can accommodate different learning styles [54]. Data visualization improves the user's ability to comprehend their progress and monitor their food intake [43]. Contents should provide realistic goals and practical strategies to initiate change in the target behaviors [18].
Among a range of technology platforms, our mobile app satisfies the need for ubiquitous technology resulting from our qualitative study because of its convenient and user-friendly interface. The widespread use of mobile technology, along with the availability of efficient mobile broadband connections, offers a distinct opportunity to develop an innovative learning method [55]. Users can learn about healthy diet, appropriate physical activity, and weight management by using short interactive videos, animations, games, or Web-based quizzes [55,56]. Mobile app has gained popularity among perinatal women [57] because of its handy features, easy usage, and multifunctional attributes. Mobile apps also offer self-regulatory features, which may promote personal awareness of health behaviors in users $[13,14]$. With regard to interactivity, users preferred flexible communication with health care professionals through the provision of Web-based discussion and access to individualized expert advice. Cloud computing offers flexible dissemination channels between health settings and health care providers [58]. Peer support is an important element of the intervention, which allows users to share their experiences, knowledge, and emotional, social, or practical support with one another [51]. Furthermore, we need to update the content and system continually to ensure that the content is updated and credible. Optimizing and maintaining user engagement remain a considerable challenge. Hence, engagement strategies are essential to app designs. These strategies include ease of use, aesthetic design, feedback function, ability to change designs to suit an individual's preference, tailored information, and distinct mobile phone features [59].

\section{Implications}

We demonstrated that a 3-step approach can be applied to develop mHELP for overweight or obese multiethnic perinatal women. Results are useful to design a culture-specific, multifaceted, and user-friendly app. The ubiquity of app facilitates the dissemination of information, supports a broad range of audience, and allows the tailoring of information and support according to users' characteristics and experiences [60]. The popularity of technological advancement can indicate a shift toward maternal empowerment within the maternity care provision [14]. Overweight and obese perinatal women can access mHELP at any time and place. Hence, mHELP can provide support for perinatal women between consultation visits, thereby reducing the number of required outpatient clinic visits [61].

\section{Limitations}

Our study presents several limitations. First, the purposive, regional, and small sample in one hospital may limit the generalizability of our findings. Second, the time to develop a 3 -step process is long, and time lags may also occur because of the changeable consumer profiles and fast-paced technological development. Third, app intervention development, including time, labor, facility, equipment, and training, is considerably resource-intensive. Thus, policy makers should consider providing financial support, manpower, protected time, and logistic support for app development. 


\section{Future Work}

This paper supports the use of a 3-step process as evidence of the usefulness of this approach. We accommodated the perspective of potential user with theoretical basis and evidence for mHELP development. Further work is needed to perform beta test in the feasibility study before RCT. In beta testing, we will evaluate the clarity of language, ease of screen navigation, technical bugs, corrupt hyperlinks, and typographical errors in various internet browsers. In addition, we will conduct a qualitative study to elicit the users' experiences after intervention. Further application and refinement will help establish evidence about the acceptability, usability, adherence, sustainability, and cost-effectiveness of mHELP. After mHELP refinement, we will evaluate its effectiveness in large and well-designed RCTs in different settings. Hence, mHELP is tailored as a culturally relevant app for obese and overweight perinatal women.

\section{Conclusions}

With the growth of smartphone devices, a series of mobile apps has been developed to provide education, information, and support concerning health problems. Theory, evidence, and user needs are vital in intervention development. The iterative process allows the incorporation of end-user feedback, theories, and systematic reviews to formulate the content, platform, and format of mHELP, which is tailored to the user's preferences. Our 3-step developmental process is a useful guide for researchers or app developers for future app development.

\section{Acknowledgments}

The authors are grateful for the generous participation of the pregnant women in this research. They also sincerely thank the assistance of the nursing staff of the National University Hospital, Singapore. The Humanities \& Social Sciences Seed Fund Grant (Reference No.: HSS Seed Funding-1/2016, WBS no: R-545-000-076-646) from National University of Singapore has supported this research.

\section{Authors' Contributions}

YL designed the research. LJC and YL collected the data. SSTHL and CC coordinated the study. YL, KLT, and WW designed interface of intervention. CT, KWO, and CC designed the content of intervention. YL and LJC analyzed and interpreted the data. YL wrote the manuscript. LJC prepared figures and tables. All authors approved the final manuscript.

\section{Conflicts of Interest}

None declared.

\section{References}

1. $\mathrm{Ng} \mathrm{M,} \mathrm{Fleming} \mathrm{T,} \mathrm{Robinson} \mathrm{M,} \mathrm{Thomson} \mathrm{B,} \mathrm{Graetz} \mathrm{N,} \mathrm{Margono} \mathrm{C,} \mathrm{et} \mathrm{al.} \mathrm{Global,} \mathrm{regional,} \mathrm{and} \mathrm{national} \mathrm{prevalence} \mathrm{of}$ overweight and obesity in children and adults during 1980-2013: a systematic analysis for the Global Burden of Disease Study 2013. Lancet 2014 Aug 30;384(9945):766-781. [doi: 10.1016/S0140-6736(14)60460-8] [Medline: 24880830]

2. MacInnis N, Woolcott CG, McDonald S, Kuhle S. Population attributable risk fractions of maternal overweight and obesity for adverse perinatal outcomes. Sci Rep 2016 Mar 10;6:22895 [FREE Full text] [doi: 10.1038/srep22895] [Medline: 26961675]

3. Vesco KK, Dietz PM, Rizzo J, Stevens VJ, Perrin NA, Bachman DJ, et al. Excessive gestational weight gain and postpartum weight retention among obese women. Obstet Gynecol 2009 Nov;114(5):1069-1075. [doi: 10.1097/AOG.0b013e3181baeacf] [Medline: 20168109]

4. Grivell RM, O'Brien CM, Dodd JM. Managing obesity in pregnancy: a change in focus from harm minimization to prevention. Semin Reprod Med 2016 Mar;34(2):e38-e46. [doi: 10.1055/s-0036-1583532] [Medline: 27144372]

5. Durst JK, Sutton AL, Cliver SP, Tita AT, Biggio JR. Impact of gestational weight gain on perinatal outcomes in obese women. Am J Perinatol 2016 Jul;33(9):849-855. [doi: 10.1055/s-0036-1579650] [Medline: 26960701]

6. Teede H, Moran L. Lifestyle factors focused on diet and physical activity: recommendations preconception and during pregnancy. Semin Reprod Med 2016 Mar;34(2):65-66. [doi: 10.1055/s-0036-1572354] [Medline: 26854710]

7. Lally P, Gardner B. Promoting habit formation. Health Psychol Rev 2013 May;7(sup1):S137-S158. [doi: 10.1080/17437199.2011.603640]

8. Muktabhant B, Lawrie TA, Lumbiganon P, Laopaiboon M. Diet or exercise, or both, for preventing excessive weight gain in pregnancy. Cochrane Database Syst Rev 2015 Jun 15(6):CD007145. [doi: 10.1002/14651858.CD007145.pub3] [Medline: 26068707]

9. Ministry of Health. Singapore: Ministry of Health; 2016. Nutrition during pregnancy: eating right for two URL: https:/ /www.healthhub.sg/live-healthy/928/pregnancy-nutrition-during-pregnancy-eating-right-for-two [accessed 2018-03-14] [WebCite Cache ID 6xvVYjONV]

10. Price BB, Amini SB, Kappeler K. Exercise in pregnancy: effect on fitness and obstetric outcomes-a randomized trial. Med Sci Sports Exerc 2012 Dec;44(12):2263-2269. [doi: 10.1249/MSS.0b013e318267ad67] [Medline: 22843114]

11. Rasmussen K, Yaktine A. Weight Gain During Pregnancy: Reexamining the Guideline. Washington, DC: The National Academies Press; 2009. 
12. Choi J, Lee JH, Vittinghoff E, Fukuoka Y. mHealth physical activity intervention: a randomized pilot study in physically inactive pregnant women. Matern Child Health J 2016 May;20(5):1091-1101. [doi: 10.1007/s10995-015-1895-7] [Medline: 26649879]

13. Flores MG, Granado-Font E, Ferré-Grau C, Montaña-Carreras X. Mobile phone apps to promote weight loss and increase physical activity: a systematic review and meta-analysis. J Med Internet Res 2015;17(11):e253 [FREE Full text] [doi: 10.2196/jmir.4836] [Medline: 26554314]

14. Silva BM, Rodrigues JJ, de la Torre Diez I, López-Coronado M, Saleem K. Mobile-health: a review of current state in 2015. J Biomed Inform 2015 Aug;56:265-272. [doi: 10.1016/j.jbi.2015.06.003] [Medline: 26071682]

15. Osma J, Barrera AZ, Ramphos E. Are pregnant and postpartum women interested in health-related apps? implications for the prevention of perinatal depression. Cyberpsychol Behav Soc Netw 2016 Jun;19(6):412-415. [doi: 10.1089/cyber.2015.0549] [Medline: 27327069]

16. Tripp N, Hainey K, Liu A, Poulton A, Peek M, Kim J, et al. An emerging model of maternity care: smartphone, midwife, doctor? Women Birth 2014 Mar;27(1):64-67. [doi: 10.1016/j.wombi.2013.11.001] [Medline: 24295598]

17. BinDhim NF, Hawkey A, Trevena L. A systematic review of quality assessment methods for smartphone health apps. Telemed J E Health 2015 Feb;21(2):97-104. [doi: 10.1089/tmj.2014.0088] [Medline: 25469795]

18. Webb TL, Joseph J, Yardley L, Michie S. Using the internet to promote health behavior change: a systematic review and meta-analysis of the impact of theoretical basis, use of behavior change techniques, and mode of delivery on efficacy. $\mathrm{J}$ Med Internet Res 2010;12(1):e4 [FREE Full text] [doi: 10.2196/jmir.1376] [Medline: 20164043]

19. Riley WT, Rivera DE, Atienza AA, Nilsen W, Allison SM, Mermelstein R. Health behavior models in the age of mobile interventions: are our theories up to the task? Transl Behav Med 2011 Mar;1(1):53-71 [FREE Full text] [doi: 10.1007/s13142-011-0021-7] [Medline: 21796270]

20. Hoffmann TC, Glasziou PP, Boutron I, Milne R, Perera R, Moher D, et al. Better reporting of interventions: template for intervention description and replication (TIDieR) checklist and guide. Br Med J 2014;348:g1687 [FREE Full text] [Medline: 24609605]

21. Dixon-Woods M, Bosk CL, Aveling EL, Goeschel CA, Pronovost PJ. Explaining Michigan: developing an ex post theory of a quality improvement program. Milbank Q 2011 Jun;89(2):167-205 [FREE Full text] [doi: 10.1111/j.1468-0009.2011.00625.x] [Medline: 21676020]

22. Agarwal S, LeFevre AE, Lee J, L'Engle K, Mehl G, Sinha C, WHO mHealth Technical Evidence Review Group. Guidelines for reporting of health interventions using mobile phones: mobile health (mHealth) evidence reporting and assessment (mERA) checklist. Br Med J 2016 Mar 17;352:i1174. [Medline: 26988021]

23. Moher D, Shamseer L, Clarke M, Ghersi D, Liberati A, Petticrew M, PRISMA-P Group. Preferred reporting items for systematic review and meta-analysis protocols (PRISMA-P) 2015 statement. Syst Rev 2015 Jan;4:1 [FREE Full text] [doi: 10.1186/2046-4053-4-1] [Medline: 25554246]

24. Berlin JA, Golub RM. Meta-analysis as evidence: building a better pyramid. J Am Med Assoc 2014 Aug 13;312(6):603-605. [doi: 10.1001/jama.2014.8167] [Medline: 25117128]

25. Schnall R, Rojas M, Bakken S, Brown W, Carballo-Dieguez A, Carry M, et al. A user-centered model for designing consumer mobile health (mHealth) applications (apps). J Biomed Inform 2016 Apr;60:243-251. [doi: 10.1016/j.jbi.2016.02.002] [Medline: 26903153]

26. Yardley L, Morrison L, Bradbury K, Muller I. The person-based approach to intervention development: application to digital health-related behavior change interventions. J Med Internet Res 2015;17(1):e30 [FREE Full text] [doi: 10.2196/jmir.4055] [Medline: 25639757]

27. Buller DB, Berwick M, Shane J, Kane I, Lantz K, Buller MK. User-centered development of a smart phone mobile application delivering personalized real-time advice on sun protection. Transl Behav Med 2013 Sep;3(3):326-334 [FREE Full text] [doi: 10.1007/s13142-013-0208-1] [Medline: 24058385]

28. LeRouge C, Ma J, Sneha S, Tolle K. User profiles and personas in the design and development of consumer health technologies. Int J Med Inform 2013 Nov;82(11):e251-e268. [doi: 10.1016/j.ijmedinf.2011.03.006] [Medline: 21481635]

29. Bandura A. Social cognitive theory of self-regulation. Organ Behav Hum Decis Process 1991 Dec;50(2):248-287. [doi: 10.1016/0749-5978(91)90022-L]

30. Kirschenbaum D. Self-regulatory failure: a review with clinical implications. Clin Psychol Rev 1987 Jan;7(1):77-104. [doi: 10.1016/0272-7358(87)90005-5]

31. Baumeister RF, Vohs KD, Tice DM. The strength model of self-control. Curr Dir Psychol Sci 2016 Jun 24;16(6):351-355. [doi: 10.1111/j.1467-8721.2007.00534.x]

32. Lau Y, Klainin-Yobas P, Htun TP, Wong SN, Tan KL, Ho-Lim ST, et al. Electronic-based lifestyle interventions in overweight or obese perinatal women: a systematic review and meta-analysis. Obes Rev 2017 Sep;18(9):1071-1087. [doi: 10.1111/obr.12557] [Medline: 28544551]

33. Albright CL, Steffen AD, Wilkens LR, White KK, Novotny R, Nigg CR, et al. Effectiveness of a 12-month randomized clinical trial to increase physical activity in multiethnic postpartum women: results from Hawaii's Nā Mikimiki Project. Prev Med 2014 Dec;69:214-223 [FREE Full text] [doi: 10.1016/j.ypmed.2014.09.019] [Medline: 25285751] 
34. Phelan S, Phipps MG, Abrams B, Darroch F, Grantham K, Schaffner A, et al. Does behavioral intervention in pregnancy reduce postpartum weight retention? Twelve-month outcomes of the Fit for Delivery randomized trial. Am J Clin Nutr 2014 Feb;99(2):302-311 [FREE Full text] [doi: 10.3945/ajcn.113.070151] [Medline: 24284438]

35. Poston L, Bell R, Croker H, Flynn AC, Godfrey KM, Goff L, UPBEAT Trial Consortium. Effect of a behavioural intervention in obese pregnant women (the UPBEAT study): a multicentre, randomised controlled trial. Lancet Diabetes Endocrinol 2015 Oct;3(10):767-777 [FREE Full text] [doi: 10.1016/S2213-8587(15)00227-2] [Medline: 26165396]

36. Fjeldsoe BS, Miller YD, Marshall AL. MobileMums: a randomized controlled trial of an SMS-based physical activity intervention. Ann Behav Med 2010 May;39(2):101-111. [doi: 10.1007/s12160-010-9170-z] [Medline: 20174902]

37. Hui AL, Ludwig S, Gardiner P, Sevenhuysen G, Murray R, Morris M, et al. Community-based exercise and dietary intervention during pregnancy: a pilot study. Can J Diabetes 2006 Jan;30(2):1-7. [doi: 10.1016/S1499-2671(06)02010-7]

38. Jackson RA, Stotland NE, Caughey AB, Gerbert B. Improving diet and exercise in pregnancy with video doctor counseling: a randomized trial. Patient Educ Couns 2011 May;83(2):203-209. [doi: 10.1016/j.pec.2010.05.019] [Medline: 21459255]

39. Hill B, McPhie S, Moran LJ, Harrison P, Huang TT, Teede H, et al. Lifestyle intervention to prevent obesity during pregnancy: implications and recommendations for research and implementation. Midwifery 2017 Jun;49:13-18. [doi: 10.1016/j.midw.2016.09.017] [Medline: 27756642]

40. Tang J, Abraham C, Greaves C, Yates T. Self-directed interventions to promote weight loss: a systematic review of reviews. J Med Internet Res 2014;16(2):e58 [FREE Full text] [doi: 10.2196/jmir.2857] [Medline: 24554464]

41. Greaves CJ, Sheppard KE, Abraham C, Hardeman W, Roden M, Evans PH, IMAGE Study Group. Systematic review of reviews of intervention components associated with increased effectiveness in dietary and physical activity interventions. BMC Public Health 2011;11:119 [FREE Full text] [doi: 10.1186/1471-2458-11-119] [Medline: 21333011]

42. Sanders JP, Loveday A, Pearson N, Edwardson C, Yates T, Biddle SJ, et al. Devices for self-monitoring sedentary time or physical activity: a scoping review. J Med Internet Res 2016 May 04;18(5):e90 [FREE Full text] [doi: 10.2196/jmir.5373] [Medline: 27145905]

43. Gemming L, Utter J, Ni Mhurchu C. Image-assisted dietary assessment: a systematic review of the evidence. J Acad Nutr Diet 2015 Jan;115(1):64-77. [doi: 10.1016/j.jand.2014.09.015] [Medline: 25441955]

44. Gunderson EP. Childbearing and obesity in women: weight before, during, and after pregnancy. Obstet Gynecol Clin North Am 2009 Jun;36(2):317-32, ix [FREE Full text] [doi: 10.1016/j.ogc.2009.04.001] [Medline: 19501316]

45. Campbell M, Fitzpatrick R, Haines A, Kinmonth AL, Sandercock P, Spiegelhalter D, et al. Framework for design and evaluation of complex interventions to improve health. Br Med J 2000 Sep 16;321(7262):694-696 [FREE Full text] [Medline: $\underline{10987780]}$

46. Braun V, Clarke V. Using thematic analysis in psychology. Qual Res Psychol 2006 Jan;3(2):77-101. [doi: 10.1191/1478088706qp063oa]

47. Hewitt-Taylor J. Use of constant comparative analysis in qualitative research. Nurs Stand 2001;15(42):39-42. [doi: 10.7748/ns2001.07.15.42.39.c3052] [Medline: 12212430]

48. Tobin GA, Begley CM. Methodological rigour within a qualitative framework. J Adv Nurs 2004 Nov;48(4):388-396. [doi: 10.1111/j.1365-2648.2004.03207.x] [Medline: 15500533 ]

49. Birt L, Scott S, Cavers D, Campbell C, Walter F. Member checking: a tool to enhance trustworthiness or merely a nod to validation? Qual Health Res 2016 Jun 22 Epub ahead of print. [doi: 10.1177/1049732316654870] [Medline: 27340178]

50. Wolf ZR. Exploring the audit trail for qualitative investigations. Nurse Educ 2003;28(4):175-178. [Medline: 12878896]

51. Kingod N, Cleal B, Wahlberg A, Husted GR. Online peer-to-peer communities in the daily lives of people with chronic illness: a qualitative systematic review. Qual Health Res 2017 Jan;27(1):89-99. [doi: 10.1177/1049732316680203] [Medline: 27956659]

52. Kelders SM, Van Gemert-Pijnen EW, Werkman A, Nijland N, Seydel ER. Effectiveness of a web-based intervention aimed at healthy dietary and physical activity behavior: a randomized controlled trial about users and usage. J Med Internet Res 2011;13(2):e32 [FREE Full text] [doi: 10.2196/jmir.1624] [Medline: 21493191]

53. Beach P. Self-directed online learning: a theoretical model for understanding elementary teachers' online learning experiences. Teach Teach Educ 2017 Jan;61:60-72 [FREE Full text] [doi: 10.1016/j.tate.2016.10.007]

54. Bert F, Gualano MR, Brusaferro S, De Vito E, de Waure C, La Torre G, et al. Pregnancy e-health: a multicenter Italian cross-sectional study on Internet use and decision-making among pregnant women. J Epidemiol Commun Health 2013 Dec 01;67(12):1013-1018. [doi: 10.1136/jech-2013-202584] [Medline: 24072743]

55. Fulantelli G, Taibi D, Arrigo M. A framework to support educational decision making in mobile learning. Comput Hum Behav 2015 Jun;47:50-59 [FREE Full text] [doi: 10.1016/j.chb.2014.05.045]

56. Shuib L, Shamshirband S, Ismail MH. A review of mobile pervasive learning: applications and issues. Comput Hum Behav 2015 May;46:239-244 [FREE Full text] [doi: 10.1016/j.chb.2015.01.002]

57. O'Higgins A, Murphy OC, Egan A, Mullaney L, Sheehan S, Turner MJ. The use of digital media by women using the maternity services in a developed country. Ir Med J 2014;107(10):313-315. [Medline: 25556255]

58. Davidesko S, Segal D, Peleg R. Online medicine for pregnant women. Int J Telemed Appl 2014;2014:379427 [FREE Full text] [doi: 10.1155/2014/379427] [Medline: 25132848] 
59. Garnett C, Crane D, West R, Brown J, Michie S. Identification of behavior change techniques and engagement strategies to design a smartphone app to reduce alcohol consumption using a formal consensus method. JMIR Mhealth Uhealth 2015;3(2):e73 [FREE Full text] [doi: 10.2196/mhealth.3895] [Medline: 26123578]

60. Tran J, Tran R, White Jr JR. Smartphone-based glucose monitors and applications in the management of diabetes: an overview of 10 salient "apps" and a novel smartphone-connected blood glucose monitor. Clin Diabet 2012 Oct 15;30(4):173-178. [doi: 10.2337/diaclin.30.4.173]

61. Pérez-Ferre N, Galindo M, Fernández MD, Velasco V, Runkle I, de la Cruz MJ, et al. The outcomes of gestational diabetes mellitus after a telecare approach are not inferior to traditional outpatient clinic visits. Int J Endocrinol 2010;2010:386941 [FREE Full text] [doi: 10.1155/2010/386941] [Medline: 20628517]

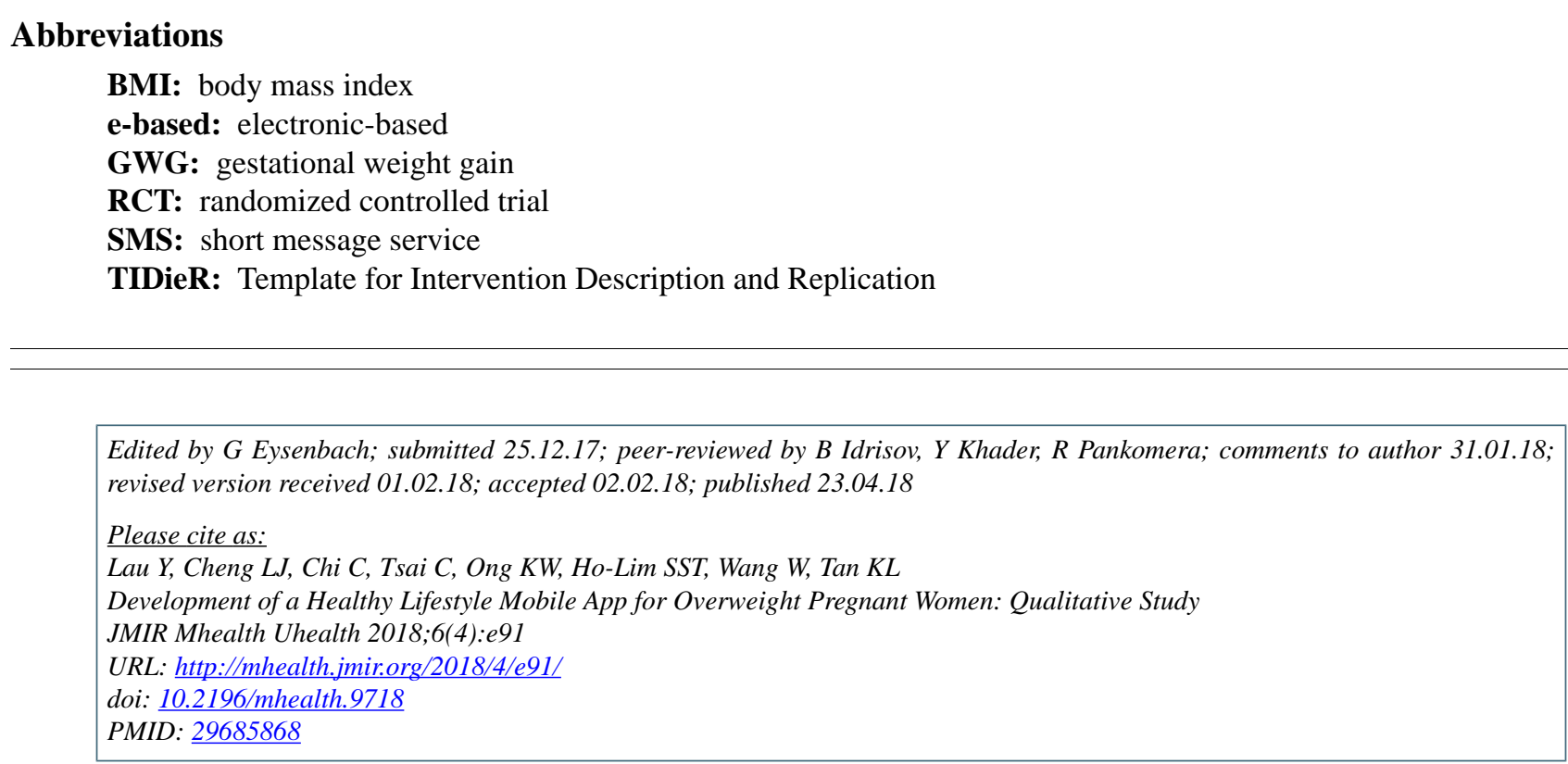

(C) Ying Lau, Ling Jie Cheng, Claudia Chi, Cammy Tsai, Kai Wen Ong, Sarah Su Tin Ho-Lim, Wei Wang, Kian-Lee Tan. Originally published in JMIR Mhealth and Uhealth (http://mhealth.jmir.org), 23.04.2018. This is an open-access article distributed under the terms of the Creative Commons Attribution License (https://creativecommons.org/licenses/by/4.0/), which permits unrestricted use, distribution, and reproduction in any medium, provided the original work, first published in JMIR mhealth and uhealth, is properly cited. The complete bibliographic information, a link to the original publication on http://mhealth.jmir.org/, as well as this copyright and license information must be included. 